Meta

Journal des tradlucteurs

Translators' Journal

\title{
Le contexte terminographique
}

\section{Bruno de Bessé}

Volume 36, numéro 1, mars 1991

La terminologie dans le monde : orientations et recherches

URI : https://id.erudit.org/iderudit/002067ar

Aller au sommaire du numéro

Éditeur(s)

Les Presses de l'Université de Montréal

\section{ISSN}

0026-0452 (imprimé)

Découvrir la revue

Citer cet article

de Bessé, B. (1991). Le contexte terminographique. Meta, 36(1), 111-120.

Ce document est protégé par la loi sur le droit d'auteur. L'utilisation des services d'Érudit (y compris la reproduction) est assujettie à sa politique d'utilisation que vous pouvez consulter en ligne.

https://apropos.erudit.org/fr/usagers/politique-dutilisation/
Cet article est diffusé et préservé par Érudit.

Érudit est un consortium interuniversitaire sans but lucratif composé de l'Université de Montréal, l'Université Laval et l'Université du Québec à Montréal. Il a pour mission la promotion et la valorisation de la recherche. https://www.erudit.org/fr/ 


\title{
LE CONTEXTE TERMINOGRAPHIQUE
}

\author{
BRUNo DE BESSÉ \\ Université de Genève, Genève, Suisse
}

\section{INTRODUCTION}

La démarche de la terminographie, et à plus forte raison celle de la terminologie, est onomasiologique, allant du concept au signe. Pour le terminographe, comme pour le terminologue, le travail consiste à délimiter, à distinguer et à définir des concepts. L'entrée terminographique n'est pas vraiment le terme, mais plutôt la réalité décrite et plus précisément sa représentation conceptuelle. La définition se fait par référence à la chose que le signe dénote, en dehors de la langue.

Mais le terme a aussi une existence dans la langue. Chaque terme se caractérise, comme chaque mot, par un fonctionnement syntaxique particulier. Son utilisation n'est pas libre. Certains éléments lexicaux font habituellement partie de son environnement. On observe par exemple la fréquence de l'association d'un terme avec certains verbes. La présence simultanée du terme et de plusieurs éléments dans le même énoncé permet de parler de cooccurrence. L'association fréquente et la proximité régulière des mêmes mots avec les mêmes termes constituent de véritables réseaux de collocations.

Les terminologues et les terminographes s'intéressent en premier lieu, et parfois un peu exclusivement, à la définition. Le contexte (et à plus forte raison la phraséologie, les collocations, les cooccurrents) est souvent considéré comme une donnée terminologique secondaire. Le plus souvent il sert soit à attester l'existence du terme, soit à l'associer à un domaine, soit encore à compléter la définition ou à pallier l'absence de définition en donnant des informations sur le concept. Le contexte n'illustre que rarement le comportement syntaxique du terme. Il conviendrait de lui donner une fonction et une dimension nouvelles, dont l'intérêt pour le traducteur, qui travaille en langue, est évident.

En effet, le traducteur, constamment à la recherche de l'expression la plus idiomatique, ne peut ignorer l'usage. Cela est vrai pour la langue commune où, par exemple, on associera le plus souvent le mot curiosité avec les verbes éveiller, exciter, piquer ou satisfaire. Mais cela vaut aussi pour les langues de spécialité où ces associations ne viennent pas toujours naturellement à l'esprit du traducteur. Ainsi les verbes augmenter, dresser ou présenter sont-ils fréquemment associés au terme budget. Il serait donc utile d'incorporer des listes de cooccurrents, accompagnés le cas échéant d'exemples d'emploi, aux fiches terminologiques traditionnelles. Ces informations permettraient au traducteur de trouver rapidement, pour chaque terme, la phraséologie appropriée et d'éviter ainsi les calques.

Sans doute n'est-il pas inutile de rappeler que les activités terminographiques ont essentiellement pour objet d'améliorer la quantité et la qualité du travail du traducteur, et pour ce faire, de lui fournir les informations les plus pertinentes. Or, pour riche qu'elle soit, la liste des informations figurant sur une fiche ne contient pas en général de véritables renseignements phraséologiques, qui sont pourtant indispensables à toute traduction idiomatique, c'est-à-dire à toute traduction de qualité. 


\section{LE CONTEXTE}

La plupart des fiches conçues et utilisées par les organismes de terminologie, et notamment celles des banques de terminologie, comportent un champ particulier réservé au contexte. Les manuels consacrent quelques lignes au contexte et distinguent habituellement plusieurs types de contextes. Mais on constate l'absence de véritable réflexion sur le sujet.

\subsection{DÉFINITION DU CONTEXTE}

On entend par contexte l'environnement linguistique d'un terme. Le contexte est constitué par l'énoncé qui entoure le terme (mots situés à proximité du terme, phrase), et qui conditionne son existence, sa forme, son fonctionnement, son sens, sa valeur et son emploi.

Le contexte a deux fonctions: il sert à éclairer le sens d'un terme et à illustrer son fonctionnement.

La définition proposée fait apparaître une certaine ambiguité quant au rôle du contexte. Cette ambivalence est confirmée par la multiplicité et la diversité des contextes.

\subsection{TYPES DE CONTEXTES}

On rencontre plusieurs types de contextes que l'on peut regrouper en deux catégories : les contextes qui renvoient au concept et les contextes liés au terme.

\subsubsection{LES CONTEXTES QUI RENVOIENT AU CONCEPT}

Les contextes qui renvoient au concept apportent des informations sur le concept, mais n'éclairent pas vraiment le fonctionnement du terme.

\subsubsection{LE CONTEXTE DÉFINITOIRE}

Le contexte définitoire contient un certain nombre d'éléments utiles et nécessaires à la description du concept, mais insuffisants pour la rédaction d'une définition. Dans ce cas le contexte pallie l'absence de définition. Cette situation peut s'expliquer par l'insuffisance de la recherche qui n'a pas permis de trouver une définition satisfaisante. Mais la présence de contextes définitoires s'explique le plus souvent par un inachèvement de la recherche.

\section{aciers}

On appelle aciers les alliages ferreux qui sont aptes au façonnage à chaud; à l' exception de certains aciers à haute teneur en chrome, ils ont une teneur en carbone égale ou inférieure à $2 \%$, teneur limite qui les sépare des fontes 1 .

Les contextes définitoires peuvent traduire une hésitation du terminographe face à une réalité nouvelle difficile à décrire, donc à définir, voire un manque d'assurance du rédacteur de la fiche.

disque optique numérique; disque digital

Le disque optique numérique ou disque digital est destiné au marché du traitement de l'information, pour le stockage de données numériques et alphanumériques, d'images de toutes informations stockées sous forme binairel.

disque optique numérique

Un disque optique numérique. Les travaux de recherches de Philips, qui ont porté leurs fruits au niveau du vidéo-disque et du compact-disc, sont aussi à la base de systèmes d'enregistrement optique de données numériques, qui permet le stockage d'un nombre considérable de données sur la surface sensible d' un disque de verre de $30 \mathrm{~cm}$ de diamètre 2 . 
Mais, dans certains cas, le contexte est le seul élément permettant de décrire le concept, par exemple quand celui-ci est défini de façon très particulière dans un texte juridique.

nouveau réservoir

Au sens du présent arrêté, on entend par nouveau réservoir tout réservoir dont l'autorisation d'exploitation au titre de la législation des installations classées intervient après la publication du présent arrêté ${ }^{3}$.

VE legs

$P H$ Les legs et les fondations non autonomes sont des patrimoines de l'État qui n'ont pas la personnalité juridique et qui lui ont été cédés par des particuliers, volontairement et pour une affectation déterminée $e^{4}$.

Dans le dernier exemple, le terminographe s'est trouvé dans la situation suivante. Le terme, qui fait l'objet de la fiche, figure dans la loi et correspond à un concept qu'il convient d'inclure dans la description du domaine et de définir. Malheureusement, la loi ne le définit pas. Le terminographe ne dispose que d'un contexte qu'il est difficile de transformer en définition. Il ne lui appartient pas en effet d'interpréter la loi et de se substituer au législateur. En pareille circonstance, l'utilisation du contexte est la seule solution acceptable, à moins d'avoir recours aux dictionnaires qui donnent des définitions provenant d'autres législations, ce qui ne manque pas de poser quelques problèmes...

Parfois, l'impossibilité de rédiger une définition en compréhension amène le terminographe à considérer comme contexte un énoncé qui contient une définition en extension peu conforme aux règles d'écriture de la définition. Les contextes suivants illustrent bien cette difficulté. Ils ne permettent pas en effet de rédiger des définitions vraiment satisfaisantes.

Canada

Le Canada comprend la masse continentale du Canada, les eaux intérieures et la mer territoriale $^{5}$.

zone à bâtir

Les zones à bâtir comprennent les terrains propres à la construction qui sont déjà largement bâtis, ou seront probablement nécessaires à la construction dans les quinze ans à venir et seront équipés dans ce laps de temps6.

produits agricoles

Sont considérés comme produits agricoles le bétail, la volaille, le lait, les légumes et les fruits - ainsi que tous leurs produits - le miel, le sirop d'érable, le tabac, les plantes textiles et fourragères, de même que les produits agricoles classés comme tels par le gouverneur en conseil pour l'application de la présente loi ${ }^{5}$.

planète

Les planètes du système solaire sont: Mercure, Vénus, Terre, Mars, Jupiter, Saturne, Uranus, Neptune, Pluton ${ }^{7}$.

Il est tout à fait possible en revanche de rédiger une définition en compréhension de planète qui soit parfaitement acceptable.

planète

Corps céleste décrivant autour du soleil une orbite elliptique peu allongée dans un plan voisin de l'écliptique ${ }^{8}$.

2.2.1.2. LE CONTEXTE CONCEPTUEL

Le contexte conceptuel associe un terme avec d'autres termes qui appartiennent à un même domaine. Il peut servir à représenter une partie de l'arbre conceptuel et indiquer 
la place qu'occupe le concept décrit dans cet ensemble. Il a tout à fait sa raison d'être dans la description terminographique d'un système conceptuel, mais serait sans doute plus à sa place dans la note.

\subsubsection{LE CONTEXTE ENCYCLOPÉDIQUE}

Le contexte encyclopédique est destiné à compléter une définition et donne des informations supplémentaires dont la présence dans la définition n'est ni utile, ni souhaitable. Les informations contenues dans ce type de contexte devraient normalement figurer dans la note.

VE osmose inverse

DF Procédé de séparation ou de concentration des corps dissous dans une solution par application d'une pression élevée (40 bars) provoquant le mouvement inverse de celui de l'osmose.

PH L'osmose inverse est utilisée notamment pour le dessalement de l'eau de mer: l'eau salée est comprimée sur des membranes hémiperméables à base d'acétate de cellulose; l'eau pure est recueillie de l'autre côté de ces membranes'.

VE actifs; éléments de l'actif

DF Ensemble des éléments du patrimoine d'une collectivité publique qui figurent à l'actif du bilan.

PH Les actifs comprennent les disponibilités, les avoirs, les placements, les actifs transitoires ainsi que les biens d'investissement, les prêts et participations permanentes, les subventions d'investissement et les autres dépenses à amortir, les avances aux Fonds des financements spéciaux et, le cas échéant, le découvert du bilan ${ }^{4}$.

\subsubsection{LE CONTEXTE MATIÈRE}

Le contexte matière fournit des indications qui permettent de rattacher le terme à un domaine particulier. Il ne se justifie pas quand la fiche comporte un champ servant à indiquer le domaine. Si l'on veut compléter l'indication du domaine, notamment quand la classification utilisée n'est pas suffisamment fine, il conviendrait de le faire dans la note.

\subsubsection{LES CONTEXTES LIÉS AU TERME}

Les contextes liés au terme n'apportent que peu d'informations sur le concept et mettent l'accent sur le terme.

\subsubsection{LE CONTEXTE LANGAGIER}

Le contexte langagier constitue une simple attestation du terme et ne comporte ni élément de définition du concept, ni indication de fonctionnement du terme. Il atteste l'existence du terme comme le font beaucoup de citations dans les dictionnaires de langue. Les informations qu'il offre sur le fonctionnement syntaxique sont la plupart du temps nulles ou insignifiantes. Son rôle consiste à prouver l'existence du terme quand cela est nécessaire.

polluant : appareil de prélèvement

Le prélèvement d'échantillons ne doit pas s'effectuer dans les tourbillons rabattants causés par des bâtiments ou par des accidents de terrain; de même, aucun obstacle ne doit limiter la quantité de polluant pouvant atteindre l'appareil de prélèvement 1 .

\subsubsection{LE CONTEXTE PHRASÉOLOGIQUE}

La méthode phraséologique consiste à présenter dans plusieurs langues des phrases parallèles, qui se correspondent sémantiquement et dont la comparaison fait apparaître les équivalents. 
FR

Un train continu à bandes à chaud comporte généralement 4 cages dégrossisseuses et 6 à 7 cages finisseuses.

EN

A continuous hot-rolled strip mill generally has 4 roughing stands and 6-7 finishing stands 1 .

Séduisante à première vue pour la traduction, cette méthode n'est pas très utilisée. Elle a d'ailleurs été abandonnée par EURODICAUTOM qui en a vite épuisé les charmes. Son principal inconvénient est de ne pas illustrer le fonctionnement réel de la langue. Le plus souvent en effet, les phrases sont des créations artificielles qui ne correspondent pas à une production linguistique spontanée. Elles ne contiennent en outre aucune information sur le concept, et sont le plus souvent des attestations forgées par leur auteur.

FR

$V E$ chemin rural: chemin forestier

PH Il y a des différences considérables entre les chemins ruraux et les chemins et sentiers d'exploitation d'une part et les chemins forestiers d'autre part.

EN

VE farm road; farm track: forest road; forest track

PH There are marked differences between farm roads / farm tracks and forest roads / forest tracks!.

Dans certains cas pourtant, leur présence peut se justifier, et se révéler utile. La comparaison des différentes versions linguistiques d'un texte, quand elles ont un statut juridique équivalent, est inévitable dans une description terminographique. C'est en particulier le cas quand le corpus de dépouillement est constitué d'accords internationaux rédigés en plusieurs langues ou de textes appartenant à la législation de pays multilingues.

\subsubsection{LE CONTEXTE LINGUISTIQUE}

Le contexte linguistique met en situation le terme, illustre son usage normal et son comportement dans la langue, présente les constructions syntaxiques les plus courantes et met en évidence les collocations les plus caractéristiques. Les bons exemples de contextes linguistiques sont difficiles à trouver. L'exemple qui suit, choisi parmi les contextes les plus parlants, montre bien les limites de la formule actuelle.

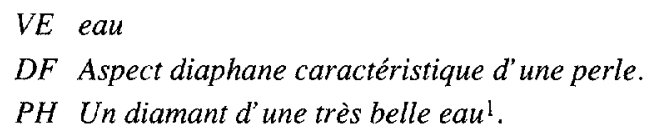

\subsubsection{LE CONTEXTE D'USAGE}

Le contexte d'usage précise l'emploi d'un terme. Il fournit des indications qui correspondent aux marques d'usage utilisées en lexicographie. Il est constitué par exemple d'un commentaire sur le fonctionnement socio-linguistique du terme. Fondamentalement métalinguistique, il n'a pas vraiment sa place dans un champ destiné à illustrer le fonctionnement syntaxique d'un terme et devrait figurer dans la note.

\subsection{PLACE DU CONTEXTE DANS LE TRAVAIL TERMINOGRAPHIQUE}

Le contexte est le point de départ de tout travail terminographique. C'est en effet le dépouillement du corpus, qui est à la fois un macrocontexte et une collection de microcontextes, qui permet de décrire un ensemble conceptuel et qui fournit les informations et les matériaux nécessaires à cette description. La multitude, la diversité et 
la richesse des contextes sont nécessaires au repérage et à la description des concepts et des termes. Ils sont indispensables à la constitution du dossier qui permettra au terminographe de rédiger une fiche, au cœur de laquelle figurera la définition, clé de voûte de la description du concept.

\subsection{CONTEXTE ET NOTE}

Le rôle de la note n'a pas encore fait l'objet d'une réflexion rigoureuse et approfondie. D'ores et déjà, sans entrer dans un sujet qui mériterait un long article à lui seul, on peut dire que la note a un rôle de complément, qu'elle peut apporter des informations soit sur le concept (informations encyclopédiques), soit sur le terme (informations sur l'usage), et qu'un certain nombre d'informations qui sont données dans le contexte devraient y figurer.

\subsection{LIMITES DU CONTEXTE}

À l'exception de certains contextes définitoires, les contextes qui renvoient au concept n'ont pas leur place dans le champ réservé au contexte. Tous les contextes recueillis lors du dépouillement et utilisés pour la rédaction de la définition n'ont pas de raison de figurer dans une fiche à l'issue de la recherche.

La présence de contextes définitoires ne peut se justifier que dans deux situations: d'une part, quand le contexte correspond à une définition en extension, qui énumère une série d'espèces; d'autre part quand les concepts sont définis de façon particulière et unique, dans un texte précis, et qu'il est impossible de rédiger une définition qui se situe à un certain niveau de généralité et d'universalité.

La plupart des contextes liés au terme ne répondent pas vraiment aux intentions de ceux qui ont conçu les modèles de fiche, et encore moins à l'attente des utilisateurs des outils terminographiques. Les attestations langagières n'ont guère d'intérêt pour l'utilisateur de la fiche. Quant aux phrases qui se correspondent dans plusieurs langues, elles ne mettent pas vraiment en parallèle des tournures idiomatiques dans plusieurs langues. Le plus souvent, ce sont des traductions plus ou moins forcées qui ne représentent pas le fonctionnement véritable de la langue. Les remarques concernant l'usage se situent au niveau métalinguistique et doivent être placées dans la note. Le contexte, apte à tous les emplois, peut aussi servir à mettre en évidence ou à attester l'existence de synonymes. Seuls devraient être retenus les contextes qui illustrent le fonctionnement du terme. Malheureusement, ce sont ceux que l'on rencontre le plus rarement.

Le plus souvent les contextes provoquent la déception et l'insatisfaction de l'utilisateur. Ce champ ne fait pas en général l'objet de soins attentifs et ne suscite pas l'intérêt du terminographe, qui a tendance à l'utiliser sans conviction et à oublier sa raison d'être, sa finalité et son rôle. L'utilisateur de son côté cache mal sa perplexité et sa déception, et n'attend pas grand-chose du contexte qui ne lui apprend rien sur le fonctionnement des termes.

\section{LA CONNAISSANCE DU FONCTIONNEMENT DES TERMES}

\subsection{LES BESOINS}

Quand le travail de rédaction de la définition est achevé, on considère que les contextes ont joué leur rôle et ont bien mérité un séjour aux archives. Mais la définition du concept, à juste titre considérée comme fondamentale, ne suffit pas toujours à l'utilisateur et en particulier au traducteur. La représentation du concept n'existe dans la 
langue que sous la forme du terme, qui, en tant que forme linguistique, appartient à un système qui fonctionne selon certaines règles proprement linguistiques, notamment morphologiques et syntaxiques.

Il faut avoir recours à la présentation des emplois réels des termes dans le discours pour en connaître le fonctionnement, et permettre ainsi à l'utilisateur d'insérer correctement les termes dans la structure de la phrase, en indiquant les exigences syntaxiques et les contraintes stylistiques de la langue. Comme l'article du dictionnaire bilingue, la fiche terminologique doit permettre à l'utilisateur de produire des énoncés.

La phraséologie propre à chaque domaine et à chaque terme, qui est la préoccupation centrale des spécialistes des langages de spécialité (LSP), fait l'objet d'une demande des utilisateurs des outils terminographiques, qu'ils soient traducteurs ou rédacteurs. Elle ne figure pas en règle générale dans les banques de terminologie et encore moins dans les dictionnaires terminographiques. Les terminographes s'intéressent essentiellement aux problèmes de dénomination, de définition et d'équivalence, ce qui incite les traducteurs par exemple à constituer des fichiers contenant les tournures ou expressions propres à certains domaines, qu'ils ne trouvent pas dans les outils terminographiques.

\subsection{L'ÉTAT DE LA RECHERCHE}

Plusieurs terminologues-terminographes semblent réfléchir au meilleur moyen de pallier l'insuffisance de ces informations. Puisqu'il s'agit du fonctionnement d'une forme linguistique dans la langue, ils se sont tournés tout naturellement vers la linguistique pour chercher des réponses à leurs questions. Dans cette perspective, trois articles ont posé en des termes voisins le problème de la présence dans la fiche de renseignements illustrant le fonctionnement des termes. Leurs auteurs se sont intéressés aux études concernant le fonctionnement des mots, effectuées sur la langue générale. Ils proposent des désignations différentes leur permettant d'identifier le besoin, de décrire le phénomène et de cerner la problématique.

\subsection{LES PHRASÉOLOGISMES}

M.C. Cormier ${ }^{9}$ parle de phraséologisme et se demande si la phraséologie est à la langue de spécialité ce que la locution ou l'expression sont à la langue générale, ce qui supposerait que le phraséologisme possède une unité de forme et de sens, qu'il s'écarte de la norme grammaticale et qu'il possède une valeur métaphorique particulière. Elle en arrive à la conclusion que le phraséologisme n'est pas vraiment assimilable à la locution.

Les exemples de phraséologismes donnés par M.C. Cormier comme définir une macro ou créer un fichier illustrent bien la signification qu'elle donne à phraséologisme.

On trouve dans la plupart des banques de nombreux exemples pouvant correspondre à cette définition.

$D E$

eine Beschwerde auf dem Dienstweg einreichen

FR introduire une réclamation par la voie hiérarchique ${ }^{1}$.

$D E$

vor dem Gerichtshof Beschwerde darüber führen, dass ein Organ der Gemeinschaft es unterlassen hat $[. .$.

FR

saisir la Cour de justice pour faire grief à l'une des institutions de la Communauté d'avoir manqué de $[\ldots]^{1}$ 
Ces vedettes, qui ne sont pas des termes, sont en général considérées comme des difficultés de traduction, et parfois regroupées dans un fichier particulier.

\subsection{LES COLLOCATIONS}

Pour $\mathrm{U}$. Heid 10 , il ne suffit pas de répertorier des termes isolés dans un dictionnaire spécialisé, mais il faut aussi tenir compte des possibilités de combinaisons de ces termes. U. Heid estime à juste titre que l'on a peu décrit les combinaisons et plus particulièrement les collocations, c'est-à-dire les éléments lexicaux qui font habituellement partie de l'environnement du terme.

\subsection{LES COOCCURRENTS}

Pour désigner la présence simultanée de plusieurs éléments dans le même énoncé, on parle également de cooccurrence et pour désigner les éléments, de cooccurrents. Pour Betty Cohen 11 le cooccurrent n'est pas une expression figée. Ce n'est pas non plus une association tout à fait libre. Il se situe, en fait, à mi-chemin. C'est une association de mots consacrée par l'usage, qui a trouvé sa place dans les expressions usuelles. Les dictionnaires de langue, comme les dictionnaires de termes, ne mentionnent pas de façon systématique et organisée les cooccurrents.

\subsection{REMARQUES TERMINOLOGIQUES}

Le concept de phraséologisme (S'agit-il d'un concept? N'est-ce pas une notion?) ne manque pas de poser quelques problèmes. $\mathrm{Il}$ rappelle la méthode phraséologique dont les faiblesses sont bien connues. En outre il se situe dans la perspective des recherches sur les langages de spécialité (LSP), qui met l'accent sur la forme linguistique, qu'elle corresponde ou non à une réalité terminologique et conceptuelle. Il semble difficile de considérer la locution verbale créer un fichier comme un terme, et de la traiter comme une vedette. La réalité conceptuelle, si elle existe dans ce cas, trouvera normalement son expression linguistique dans l'un des termes suivants: création d'un fichier, création de fichier ou création fichier, qui constituera l'entrée de la fiche.

Quant au terme collocation, il semble le plus satisfaisant pour désigner la présence régulière et fréquente, autour du terme, de plusieurs éléments lexicaux qui lui sont associés de façon particulière, et dont l'utilisation n'est pas libre. Ce terme est préférable à cooccurrence pour caractériser le fait lui-même.

On réservera le terme cooccurrent à la désignation des éléments présents autour du terme qui répondent à la définition de la collocation donnée ci-dessus.

\section{DESCRIPTION DU FONCTIONNEMENT LINGUISTIQUE DES TERMES}

La plupart du temps, les cooccurrents ne sont pas des termes. Certes, des termes peuvent faire partie de la liste des cooccurrents. Mais le plus souvent les cooccurrents sont des mots ou des syntagmes de la langue générale, qui sont régulièrement associés aux termes, qui prennent à cette occasion un sens nouveau et constituent une forme linguistique particulière.

Les cooccurrents, dans la mesure où ils ne sont pas des termes et où ils ne correspondent pas à des concepts, ne peuvent constituer des entrées. Étant donné la grande quantité de cooccurrents susceptibles d'être associés à un terme, les cooccurrents ne peuvent pas, en interrogation normale, servir de clés d'accès à la fiche. Le terme vedette, et le cas échéant ses synonymes, doit rester la clé d'accès privilégiée à la fiche qui correspond à une unité conceptuelle, même si elle contient un faisceau de relations et de collocations linguistiques. 
La nécessité d'intégrer les informations concernant le fonctionnement des termes dans la description terminographique et de prendre en compte les cooccurrents, amène à proposer une méthode de travail pour la description et le traitement de ces données.

\subsection{LA DÉLIMITATION DES COOCCURRENTS}

La recherche des cooccurrents se fait naturellement avec les sources du corpus constitué pour le travail terminographique. Inévitablement se pose à cette occasion la question de la délimitation des unités terminologiques. Pour la description de la réalité conceptuelle, ce sont les déterminants essentiels qui retiennent l'attention, dans la mesure où ils correspondent aux caractères du concept. En revanche, quand il s'agit de rechercher les cooccurrents, le terminographe ne s'intéresse qu'aux déterminants accidentels, parmi lesquels il lui appartient de choisir ceux qui sont des cooccurrents.

Le choix des cooccurrents dépend non seulement du caractère particulier de leur association avec le terme, mais aussi de la fréquence de leur apparition. Certes l'association de cooccurrents à un terme ne correspond pas nécessairement à une forme ou à une structure lexicalisée, mais elle n'est pas totalement libre pour autant. C'est seulement quand il constate l'usage courant et usuel d'une certaine combinaison lexicale, que le terminographe peut conclure à l'existence d'une collocation et à la présence d'un cooccurrent.

\subsection{LES CATÉGORIES GRAMMATICALES}

Toutes les statistiques sur la répartition des termes dans les différentes catégories grammaticales donnent les mêmes résultats: $95 \%$ des termes sont des noms, 3 à $4 \%$ des verbes et 1 à $2 \%$ des adjectifs. Le nom est l'objet même de la terminologie 12 . Il n'est donc pas surprenant que la très grande majorité des entrées d'un outil terminographique soit constituée de substantifs. Naturellement cette prédominance, pour ne pas dire ce monopole, est étroitement liée au caractère dénominatif du terme. C'est donc autour de cette catégorie que doivent être organisées la recherche et la présentation des cooccurrents.

Le choix des adjectifs se fait après avoir distingué les adjectifs qualificatifs et les adjectifs de relation. La liste des adjectifs qualificatifs est ouverte, et leur intérêt plutôt limité. Quant aux adjectifs de relation, ils font le plus souvent partie des éléments qui servent à constituer le terme.

Incontestablement, ce sont les verbes qui représentent les cooccurrents les plus intéressants et surtout les plus utiles. C'est autour d'eux que se fait l'insertion dans la langue des termes qui ne peuvent fonctionner qu'en leur présence. Certes, le nombre de verbes appartenant aux terminologies est extrêmement limité, mais en revanche un grand nombre de verbes de la langue générale permettent le fonctionnement des termes. Ce sont ces verbes qu'il convient de repérer et de faire figurer dans la liste des cooccurrents.

\subsection{EXEMPLE DE DESCRIPTION DE FONCTIONNEMENT D'UN TERME}

Dans la fiche, les cooccurrents figurent dans le champ réservé au contexte et sont regroupés par catégories grammaticales.

Il n'est pas possible d'aller aussi loin dans l'analyse du fonctionnement des termes et dans la présentation des cooccurrents que Betty Cohen, qui propose une véritable carte du fonctionnement des termes dans son ouvrage ${ }^{13}$. Il semble difficile en effet, faute de place, de présenter les cooccurrents autrement que sous la forme d'une liste.

budget

DF/DEF Acte annuel par lequel sont prévues et autorisées les recettes et les dépenses de l'État ou d" une entreprise commerciale. 
PH/CONT v.(sujet): équilibrer, déséquilibrer; v.(objet): augmenter, diminuer, dresser, émarger au, établir, exécuter, figurer au, grever, inscrire au, porter au, préparer, présenter, puiser dans, réduire, voter; adj.: considérable, élevé, énorme, en déséquilibre, en équilibre, en excédent, excédentaire, gros, petit;

n.: augmentation, compression, diminution, établissement, hausse, majoration, préparation, réduction ${ }^{13}$.

Dans l'exemple qui précède, l'ensemble de la fiche occupe 500 caractères. L'espace est utilisé de la manière suivante: 6 caractères pour la vedette, 117 caractères pour la définition, 377 caractères pour le contexte.

\section{CONCLUSION}

Assurément, toutes les possibilités offertes par le champ réservé au contexte n'ont pas encore été explorées. D'ores et déjà, en dehors des exceptions déjà signalées, il conviendrait de ne plus utiliser ce champ à d'autres fins que celles pour lesquelles il a été prévu. Il doit essentiellement servir à montrer le fonctionnement des termes dans la langue. La présence d'une liste de cooccurrents en constitue incontestablement une bonne illustration, qu'il conviendrait d'expérimenter systématiquement dans un domaine.

Mais il faudrait sans doute examiner d'autres possibilités. La terminographie pourrait utilement s'inspirer de certaines méthodes lexicographiques.

L'intérêt récent pour la terminographie de certains linguistes, qui orientent leurs recherches sur l'observation du discours des langages de spécialité, est susceptible d'apporter quelques enrichissements. Mais l'étude du discours scientifique ou technique, même si elle peut constituer un apport important pour le terminographe, n'en reste pas moins extérieure à son objet. Il convient en effet de ne pas oublier, ce qui est trop souvent le cas, que la démarche terminographique est onomasiologique, même si le terminographe effectue sa description à partir d'énoncés. Quelle que soit l'utilité des analyses de discours pour le terminographe, il doit partir des choses et des objets à nommer pour aller aux signes. C'est seulement après avoir réalisé la délimitation et la description des concepts, qu'il peut, pour répondre à l'attente des langagiers, rechercher toutes les informations utiles sur le fonctionnement des termes.

\section{NOTES}

1. EURODICAUTOM: Banque de terminologie de la Commission des Communautés européennes.

2. TERMIUM: Banque de terminologie du Secrétariat d'État du Canada.

3. Arrêté du 4 septembre 1986 relatif à la réduction des émissions atmosphériques d'hydrocarbures provenant des activités de stockage. Journal officiel de la République Française, 20 novembre 1986, p. 14355

4. LINGUA-PC: Banque de terminologie du canton de Berne.

5. Cyrille Goulet (1989): Recueil des définitions des lois fédérales, Bulletin de terminologie 153, ministère des Approvisionnements et Services Canada.

6. Loi fédérale sur l'aménagement du territoire (LAT) du 22 juin 1979.

7. Principes et méthodes de la terminologie (1987), Norme internationale ISO 704, Organisation internationale de normalisation.

8. Le Grand Robert de la langue française (1985), Dictionnaire alphabétique et analogique de la langue française.

9. Monique C. Cormier (1989): «La terminologie: du terme au texte», Terminologie diachronique, Conseil international de la langue française.

10. U. Heid (1989): «Attentes des terminologues à l'égard des programmes d'élaboration des dictionnaires», Terminologie diachronique, Conseil international de la langue française.

11. Betty Cohen (1987): «Vous avez dit... cooccurrent?», Terminogramme, 41-42, février, Office de la langue française, Gouvernement du Québec.

12. Alain Rey (1979): La terminologie: noms et notions, Que sais-je?, Presses Universitaires de France.

13. Betty Cohen (1986): Lexique des cooccurrents, Bourse - Conjoncture économique, Linguatech. 